\title{
Glioblastoma Recurrence after Cediranib Therapy in Patients: Lack of "Rebound" Revascularization as Mode of Escape
}

\author{
Emmanuelle di Tomaso ${ }^{1,6}$, Matija Snuderl 2,6 , Walid S. Kamoun ${ }^{1,6}$, Dan G. Duda ${ }^{1}$, Pavan \\ K. Auluck ${ }^{2}$, Ladan Fazlollahi ${ }^{2}$, Ovidiu C. Andronesi ${ }^{3}$, Matthew P. Frosch ${ }^{2}$, Patrick Y. Wen ${ }^{4}$, \\ Scott R. Plotkin ${ }^{5}$, E. Tessa Hedley-Whyte ${ }^{2}$, A. Gregory Sorensen ${ }^{3}$, Tracy T. Batchelor ${ }^{5}$, and \\ Rakesh K. Jain ${ }^{1, *}$ \\ ${ }^{1}$ Department of Radiation Oncology, Massachusetts General Hospital, Harvard Medical School, \\ Boston, MA 02114, USA \\ 2 Department of Pathology (Neuropathology), Massachusetts General Hospital, Harvard Medical \\ School, Boston, MA 02114, USA \\ ${ }^{3}$ Martinos Center for Biomedical Imaging, Massachusetts General Hospital \& Massachusetts \\ Institute of Technology, Harvard Medical School, Boston, MA 02114, USA \\ 4 Department of Adult Oncology, Dana-Farber Cancer Institute, Harvard Medical School, Boston, \\ MA 02114, USA \\ ${ }^{5}$ Stephen E. \& Catherine Pappas Center for Neuro-Oncology, Department of Neurology, \\ Massachusetts General Hospital, Harvard Medical School, Boston, MA 02114, USA
}

\section{Abstract}

Recurrent glioblastomas (rGBM) invariably relapse after initial response to anti-VEGF therapy. There are two prevailing hypotheses on how these tumors escape antiangiogenic therapy: switch to VEGF-independent angiogenic pathways and vessel co-option. However, direct evidence in rGBM patients is lacking. Thus, we compared molecular, cellular and vascular parameters in autopsy tissues from five rGBM patients who had been treated with the pan-VEGF receptor tyrosine kinase inhibitor cediranib versus seven patients who received no therapy or chemoradiation but no antiangiogenic agents. After cediranib treatment, endothelial proliferation and glomeruloid vessels were decreased, and vessel diameters and perimeters were reduced to levels comparable to the unaffected contralateral brain hemisphere. In addition, tumor endothelial cells expressed molecular markers specific to the blood-brain barrier, indicative of a lack of revascularization despite the discontinuation of therapy. Surprisingly, in cediranib-treated GBM cellular density in the central area of the tumor was lower than in control cases and gradually decreased towards the infiltrating edge, indicative of a change in growth pattern of rGBMs after cediranib treatment, unlike that after chemo-radiation. Finally, cediranib treated GBMs showed high levels of PDGF-C and c-Met expression and infiltration by myeloid cells, which may potentially contribute to resistance to antiVEGF therapy. In summary, we show that rGBMs switch their growth pattern after anti-VEGF therapy - characterized by lower tumor cellularity in the central area, decreased pseudopalisading necrosis and blood vessels with normal molecular expression and morphology without a second wave of angiogenesis.

\footnotetext{
"Corresponding author: Rakesh K. Jain, PhD, Steele Laboratory, COX-734, Massachusetts General Hospital, 100, Blossom Street, Boston, MA 02114, Ph: 617726 4083, Fax: 617724 1819, Jain@ steele.mgh.harvard.edu.

${ }^{6}$ These authors contributed equally to this study.
} 


\section{INTRODUCTION}

Abundant angiogenesis with microvascular proliferation and tumor necrosis caused by severe hypoxia are diagnostic criteria for glioblastoma (GBM)(1-4). GBM vessels are glomeruloid, dilated and tortuous, with abnormalities in pericyte coverage and basement membrane, which leads to abnormal vascular function (e.g., increased permeability) $\left(2^{,} 5^{-7}\right)$. GBM angiogenesis is driven mainly by vascular endothelial growth factor A (VEGF-A, referred to as VEGF hereonwards) signaling via its tyrosine kinase receptor VEGFR2/KDR $\left(8^{-} 10\right)$. Blockade of VEGF with bevacizumab (a VEGF-specific antibody, Genentech) or of VEGF receptors with the pan-VEGFR tyrosine kinase inhibitor cediranib (AstraZeneca) was associated with rates of objective radiographic responses in phase II trials in patients with recurrent GBM (rGBM) $(11,12)$.

In both preclinical and clinical settings, the benefits of cediranib monotherapy for gliomas are typically transient and followed by an apparent increase in tumor burden $(11,13)$. In addition, imaging studies suggest that GBM progression may not be visible on contrastenhanced MRI $(14,15)$. Moreover, preclinical studies suggest a "wave" or tumor revascularization after discontinuation of anti-VEGF therapy, which might accelerate tumor relapse $(16,17)$.

To gain insight into the changes that occur in rGBMs after anti-VEGF therapy, we analyzed brain tissues obtained at autopsy. We studied the central area versus the infiltrating edge of rGBM tissues from patients treated with cediranib at recurrence after surgery, chemotherapy and radiation. As a control, we studied in a similar manner the autopsy tissues from a group of rGBM patients who received either no treatment, or surgery and/or chemotherapy and radiation. In addition, we explored the changes in molecular, cellular, morphological profiles and microenvironmental factors that might be responsible for diffuse infiltrating behavior and resistance to cediranib therapy.

\section{MATERIALS AND METHODS}

\section{Tissue specimens}

Formalin fixed, paraffin embedded (FFPE) samples from five patients with rGBM—enrolled in a phase II clinical trial of cediranib $(11,13)$ - underwent postmortem examination. These samples were compared to (i) the initial diagnostic paraffin blocks (available from $4 / 5$ rGBM patients) prior to cediranib therapy; and (ii) seven autopsy samples from GBM patients who had received either no treatment $(n=1)$, or chemo- and radiation therapy $(n=6)$. All samples were obtained from the Neuropathology Laboratory at Massachusetts General Hospital after obtaining informed consent and Institutional Review Board (IRB) approval.

\section{Histological analysis}

For all analyses, tissues were selected to contain macroscopically identifiable rGBM and its infiltrative edge in the same block. These samples were fixed in formalin, embedded in paraffin (FFPE) and stained with hematoxylin and eosin (H\&E). In this study, the end of the block (section) that contains tumor with no recognizable normal intervening brain structure is referred to as the "central area" and the "infiltrative area" is the opposite end of the block and includes identifiable brain structures and infiltrating glioma. Each autopsy (average 20 blocks per case) was reviewed by two neuropathologists (MS, PKA) to ensure that blocks selected for the study are morphologically representative of the entire tumor. Samples were stained with standard hematoxylin and eosin (H\&E). The entire H\&E slide was scanned using a Scanscope ${ }^{\mathrm{TM}}$ scanner (Aperio Technologies, Vista, CA). Regions of interest were selected at the interface of tumor center and infiltrative edge. Cellular density was estimated as the fraction of surface area occupied by tumor nuclei (highlighted by hematoxylin) in the 
central and the infiltrative regions using an in-house threshold based segmentation of the nuclei in H\&E images using Matlab software (The MathWorks, Natick, MA). Foci of pseudopalisading necrosis i.e.: dense accumulation of tumor nuclei surrounding foci of necrosis and used as diagnostic criteria of glioblastoma by WHO classification (18) were counted in 30 high power fields ( $400 \times$ magnification) in the central area of the tumor.

\section{Immunohistochemical, immunofluorescence and Fluorescence in Situ Hybridization analysis}

Five micron-thick FFPE sections were immunostained following manufacturers' recommendations and standard protocols with antibodies against the following antigens: CD31, Ki67 (both prediluted), collagen IV (1:300) and transferrin receptor (CD71, 1:100) (all Dako, Carpinteria, CA); $\alpha$ SMA (1:100)(Sigma, St. Louis, MO); VEGFR2 (1:250), PDGFR $\alpha$ (1:100) and PDGFR $\beta$ (1:100) (all Cell Signaling, Beverly, MA); CD68 (1:100) (Thermo Fischer Scientific, Fremont, CA); nestin (Abcam, 1:500), SDF1 $\alpha$ 1:40)(Biovision, Mountain View, CA); CXCR4 (1:200) and PDGF-C (1:100)(R\&D Systems, Minneapolis, MN); and MDR1 (1:40)(Abcam, Cambridge, MA). For detection of apoptosis we used the ApopTag kit (Millipore, Billerica, MA) following manufacturer's instructions. Double Immunofluorescence (IF) staining was performed for nestin with CD34 (Abcam EP373Y, dilution 1:100) and for PDGFR $\beta$ (Cell signaling 3169, dilution 1:100) with CD34 (Becton Dickinson, clone MY10, dilution 1: 50). Following secondary antibodies were used: Cy5conjugated goat anti-mouse (Invitrogen Alexa Fluor 647, AF-647, dilution 1:50) for nestin, FITC-conjugated goat anti-rabbit (Vector FI-1000, dilution 1:50) for CD34, FITCconjugated goat anti-rabbit (Vector FI-1000, dilution 1:50) for PDGFR $\beta$ and Cy5conjugated goat anti-mouse (AF-647, dilution 1:50) for CD34. Slides were denatured with EGFR probe according to a standard Fluorescence in situ Hybridization (FISH) protocol.

Semi-quantitative analysis of all immunohistochemical stains was performed by two investigators (EdT and MS) who independently scored the intensity of staining of all proteins in tumor cells and endothelial cells using a scale from 0 (no staining) to 3 (strong staining). Microvessel density (MVD), diameter, perimeter, and the surface covered by the vascular space was estimated in at least five random fields of central or infiltrative area or normal tissue on CD31 stained sections using a customized analysis software tool compatible with Image $\mathbf{J}$ (http://rsb.info.nih.gov/ij/)(100 vessels at 200x magnification per section). PDGFR $\beta$ expression in endothelial cells was evaluated to detect the "ectopic" expression of this marker in endothelial cells of GBM or other cancers $(13,19)$. Collagen IV labeling was used to quantify the basement membrane thickness (which is abnormally thick in GBMs (6)) using a segmentation algorithm (Matlab). The profile around the vessels was fit to an exponential decay function (1):

$$
(\mathrm{F}=\mathrm{Ae}-\mathrm{x} / \mathrm{L}+\mathrm{C})
$$

where $\mathrm{F}=$ fraction of collagen IV positive area at $\times$ distance $(1$ to $10 \mu \mathrm{m})$ from the vessels, and $\mathrm{L}=$ characteristic length, which correlates with basement membrane thickness. A total of 1000 cells were counted in the areas of the highest Ki-67 expression or apoptosis and indices were calculated as a percentage of positive cells.

\section{Statistical analysis}

Data are expressed as mean \pm standard error of the mean. The principal statistical test was the t-test (two-tailed with unequal variance). We considered a $p$ value of less than 0.05 to be statistically significant. 


\section{RESULTS}

\section{Patient characteristics and response to cediranib treatment}

All five rGBM patients studied received cediranib (starting dose of $45 \mathrm{mg} / \mathrm{kg} /$ day) for at least 2 cycles (range 56-232 days). Cediranib showed some radiographic activity in all of these patients: four showed partial response (P1, P2, P4 and P5) and one a stable disease (P3) based on MRI performed at 28 days (Table 1 and Supplementary Figure S1). At the "end-of-study" MRI scan when compared to day $28 \mathrm{MRI}$, two patients showed stable disease by imaging but were progressing clinically (P1 and P4), two patients (P2 and P5) showed increased tumor volume by T1 post contrast MRI, and three patients (P1, P2 and P4) showed significantly increased FLAIR signal suggestive of infiltrating disease (Table 1 and Supplementary Figure S1). Four of the five patients received no other treatment after cediranib discontinuation. One patient (P2) received further anti-VEGF treatment with bevacizumab ( 3 cycles) and CPT-11 (irinotecan, 1 cycle). Examination of the specimen from this patient showed a similar pattern to the other four, thus data from all five patients are presented. The five rGBM patients had a total survival of 161, 259, 175, 186 and 226 days respectively, measured from the time of the first cediranib dose. Of interest, the median overall survival (OS) for the 31 patients enrolled in the phase 2 study was 227 days (177293 days)(11). Clinicopathological data of control cases are summarized in Table 2.

\section{Cediranib-treated rGBMs contain structurally normal brain vessels}

The analysis of rGBM autopsy specimens offered several lines of evidence that rGBM vessels in cediranib-treated patients resembled the normal brain vessels. First, microvascular proliferation and glomeruloid vessels, which are diagnostic features of GBM, were virtually undetectable in autopsy tissues from the patients treated with cediranib (Figures 1 and 2). As expected, these features were abundantly present in all initial biopsies prior to cediranib therapy in study subjects as well as in control autopsies. Second, the diameter and perimeter of the rGBM vessels in central areas of the tumors were significantly lower in cediranibtreated autopsy specimens compared to control specimens ( $p<0.05$, Figure 1$)$ as well as those from the patient's initial diagnostic GBM biopsy sample (data not shown). The diameter and perimeter of the vessels in the infiltrative edge of GBMs were comparable between the two groups, and close to those of vessels of the normal uninvolved brain (Figure 1). Third, the thickness of basement membrane components—as defined by collagen IV immunostaining - was also significantly reduced in the central areas of the cediranibtreated compared to control patients ( $p<0.05$, Figure 1). Overall, central areas of control cases show broad variability of vascular parameters. In contrast, tumor vascular parameters in cediranib treated patients are relatively uniform. The presence of structurally normal vessels in the central area of cediranib-treated rGBMs might represent an increased infiltration by the tumors of brain tissue and/or increased co-option of normal vessels.

Collectively, these morphometric analyses indicate that vessels within cediranib treated tumors lack characteristics of abnormal GBM vasculature despite cessation of the antiangiogenic treatment.

\section{Cediranib treatment decreases cellular density of rGBM}

The rGBMs of patients who had not undergone antiangiogenic therapy showed marked variability in nuclear density within the central area ranging between $2-12 \times 10^{3}$ nuclei/ $\mathrm{mm}^{2}$. The cellular density in the central area of GBMs in cediranib treated patients was lower and ranged between $2-5 \times 10^{3}$ cells $/ \mathrm{mm}^{2}$; however this did not reach statistical significance. In the control cases, the density decreased sharply with transition into the infiltrative area which showed narrower range of cell density. In contrast, the cediranib treated patients showed cellular densities in the infiltrative edge that were relatively similar 
to those in the central area (Figure 2). Within $1 \mathrm{~mm}$ around the tumor edge $( \pm 0.5 \mathrm{~mm})$, mean nuclear density in control cases rapidly dropped from $5301 \pm 979$ to $2838 \pm 404$ nuclei $/ \mathrm{mm}^{2}$ while mean nuclear density of cediranib-treated cases was $3202 \pm 302$ nuclei $/ \mathrm{mm}^{2}$ in the central area compared to $2326 \pm 271$ nuclei $/ \mathrm{mm}^{2}$ in the infiltrating edge (Figure 2). Interestingly, one patient with the longest interval between the last dose of cediranib and death (P5; 105 days) has the cellular density curve pattern similar to the control cases while patients with shorter interval between the last dose of cediranib and death (P1, P3, P4; 27, 16 and 42 days, respectively) and the patient who received bevacizumab until his death (P2) showed relatively flat curves.

There was no difference in proliferation or apoptosis both in the central area and infiltrating edge between cediranib-treated and control GBMs (Figure 2). Control cases showed significantly higher number of foci with classical pseudopallisading necrosis than cediranib treated cases (p-value $<0.001$, Figure 2). Therefore, anti-VEGF therapy appears to decrease cellular density in the central area but not the infiltrative edge. Since the proliferation and apoptosis rates and necrosis are not increased after cediranib therapy, this indicates either increased infiltration into the normal brain or that cediranib had direct anti-tumor effect leading to decrease of cellular density, which was not restored after discontinuation of the treatment.

\section{Molecular changes in endothelial cells in cediranib-treated rGBM}

In four out of five cediranib-treated autopsy specimens PDGFR $\alpha$ and PDGFR $\beta$ became undetectable in endothelial cells and in one case PDGFR $\beta$ expression was detectable only in a fraction of endothelial cells in the central area (Figure 1D). Specificity of PDGFR $\beta$ expression by endothelial cells was confirmed by double immunohistochemical staining and double immunofluorescent staining using the endothelial markers CD31 and CD34, respectively, and FISH for EGFR in GBM cells (Supplementary Figure S2). In addition, VEGFR2 immunoreactivity decreased from a staining intensity of the $3+$ (scored in the original biopsies) to an intensity of $1+$ (data not shown). All control specimens displayed a strong expression of all three receptors. Of particular interest, these changes in endothelial phenotype were accompanied by CD71 expression (Transferrin receptor, a marker of a functional blood-brain-barrier) supporting the decreased permeability detected by functional MRI. As expected, in the control specimens CD71 was greatly reduced in the central area of the tumor. Vessel maturation markers -angiopoietin 2 and Tie2- were quantified and found to be generally expressed by the endothelial cells in vessels in central and infiltrative areas and to a lesser extent in the normal brain tissue in both cediranib-treated and control specimen. Expression of MDR, a marker of multidrug resistance was not changed after cediranib (Supplementary Table S1).

\section{Cediranib-treated GBMs show high levels of PDGF-C, c-Met and tumor-infiltrating myeloid cells}

While the number of $\mathrm{CD}^{+} 8^{+}$tumor infiltrating myeloid cells in the infiltrating edge was comparable between control and cediraninb-treated rGBMs, two of five cediranib treated patients showed high numbers of $\mathrm{CD}^{+} 8^{+}$cells in the central area of the tumor (P1 and P3) (Figure 3A and B). The rGBMs of these two patients progressed rapidly and led to rapid death. Cediranib-treated and control tumor cells showed no difference in expression of SDF1 $\alpha$ or CXCR4 in the central or infiltrating areas (Figure 3C). However, in cediranib treated tumors, CXCR4 strongly positive myeloid cells were distributed in the similar pattern as CD68+ cells (Figure 3D).

In addition, in the rGBMs from cediranib-treated patients, PDGF-C was expressed at variable levels in all cases examined, both in initial biopsy and autopsy samples. PDGF-C 
was focally expressed also in control cases without cediranib therapy. Finally, cMet expression was detectable in all the rGBM specimens examined. Both tumor cells in the central area of cediranib treated patients as well as infiltrating cells showed expresion of cMet (Figure 4A). A trend towards higher level of expression of cMet and higher number of positive cells was observed for the cediranib-treated specimens when compared to the control specimens, consistent with the potential role of the HGF/c-Met pathway in glioma progression (20). Finally, we measured the expression of the developmental intermediate filament, nestin. Tumor cells and endothelial cells in the central areas were similarly positive for nestin in cediranib-treated and untreated tumors. Interestingly, endothelial cells in the contralateral, uninvolved part of the brain were strongly positive for nestin in cediranibtreated patients but not in control brain tissues (Figure 4B). Expression of nestin by endothelial cells was confirmed by double immunohistochemical staining and double immunofluorescent staining with the endothelial markers CD31 and CD34, respectively (Supplementary Figure S2).

\section{DISCUSSION}

Pro-infiltrating consequences of antiangiogenic therapy have been suggested in a number of preclinical studies of GBM $(21,22)$. Rubenstein et al showed in animal models that blocking VEGF signaling by an antibody increases co-option and growth of satellite tumors (21), and Du et al. showed that ablation of hypoxia-inducible factor 1 alpha (HIF1 $\alpha)$ signaling leads to co-option and deep invasion of gliomas in mice (23). Casanovas et al have shown revascularization and increased invasiveness after anti-VEGFR2 antibody therapy (24). Recently, Paez-Ribes et al. have demonstrated that orthotopically implanted GBM cells under a VEGFR-selective kinase inhibitor treatment as well as VEGF-/- GBM cells show significantly increased invasion (25). While our results are consistent with the "switch" to a more invasive growth of human rGBMs after VEGF blockade, we found no evidence for a "rebound" revascularization.

Although MRI findings in patients have suggested increased invasion as an adaptive response to bevacizumab $\left(15,26^{-} 28\right)$ and cediranib $(13,14)$, the effects of antiangiogenic monotherapy on rGBM vasculature in patients remain largely unknown. de Groot et al showed in biopsies of patients with rGBM after bevacizumab therapy that abnormal FLAIR areas contained glioma cells diffusely infiltrating with a lack of glomeruloid vascular proliferation (15). Our data did not show evidence of a switch into alternative proangiogenic pathway as an escape mechanism of recurrence under antiangiogenic therapy and despite the significant time interval between the last dose of cediranib and death, vascular morphology within the central tumor was comparable with those of a normal brain in terms of vascular density, diameter, basement membrane thickness and expression of PDGFR $\alpha$, PDGFR $\beta$, CD71, VEGFR-2, and Tie2. Furthermore, we observed decrease of cell density in the central area of the tumor, despite proliferation and apoptotic rates being similar to the tumors without antiangiogenic therapy. The striking decrease of pseudopalisading necrosis after cediranib therapy might be explained by vascular normalization after initial treatment diminishing hypoxic events leading to this characteristic type of GBM necrosis (13).

The molecular pathways that drive GBM progression through anti-VEGF therapy in patients remain largely unknown. We detected PDGF-C and c-Met as two possible candidates. We also detected nestin expression in endothelial cells in both tumor and contralateral brain vessels in patients treated with cediranib. Nestin is a marker of undifferentiated cells. This novel finding could imply that sustained interruption of the VEGF signaling might globally change the phenotype of all endothelial cells. Further studies need to be undertaken in order to explore this potential relatioship between VEGF signaling and developmental cytoskeletal proteins. Resistance to anti-VEGF therapy has also been linked to myeloid cell infiltration in 
preclinical models of solid tumors $(23,29)$, and in lymphomas in patients (30). Our data suggest that CXCR4 positive tumor associated macrophages infiltrate the tumor, possibly in response to transient increase in SDF1 $\alpha$ which correlated with rGBM progression during cediranib therapy (13). However, our analyses did not show a significant difference in tissue SDF1 $\alpha$ expression between cediranib-treated and control GBMs at the time of autopsy. Despite providing direct evidence for significant structural changes in rGBMs after antiangiogenic therapy, this study has certain limitations. The small number of autopsy samples and the natural heterogeneity of the disease warrants further studies to confirm these findings. Our study also emphasizes the importance of autopsy evaluation and brain banking as the ultimate clinicopathological correlation of a drug effect as well as a crucial step in connecting the clinical and pre-clinical studies.

In summary, our study provides first in-human morphological evidence that anti-VEGF treatment changes the growth pattern of rGBMs in patients with decreased microvascular proliferation, loss of pseudopalisading necrosis and diffuse spread into the adjacent normal brain (Figure 4). Our results show that instead of switching to alternative angiogenesis pathways, rGBMs exhibit a more infiltrative phenotype after antiangiogenic therapy.

\section{Supplementary Material}

Refer to Web version on PubMed Central for supplementary material.

\section{Acknowledgments}

This study was partially supported by grants from NIH (P01-CA80124, R01-CA115767, R21-CA117079, K24CA125440, R01-CA129371, P41-RR014075, M01-RR-01066 and Federal Share/NCI Proton Beam Program Income), and from Harvard Clinical and Translational Science Center GCRC, National Foundation for Cancer Research and the Montesi Family Research Fund.

We thank Dr. Timothy Padera and Stephen Conley for their help during the manuscript preparation.

We thank the Montesi Family for their generous support of this research project.

\section{References}

1. Jain RK, di Tomaso E, Duda DG, Loeffler JS, Sorensen AG, Batchelor TT. Angiogenesis in brain tumours. Nature reviews. 2007; 8(8):610-22.

2. Plate KH, Mennel HD. Vascular morphology and angiogenesis in glial tumors. Exp Toxicol Pathol. 1995; 47(2-3):89-94. [PubMed: 7580112]

3. Rampling R, Cruickshank G, Lewis A, Fitzsimmon S, Workman P. Direct measurment of PO2 distribution and bioreductive enzymes in human malignant brain tumors. Int J Radiat Oncol Biol Phys. 1994; 29(3):427-31. [PubMed: 8005794]

4. Valk PE, Mathis CA, Prados MD, Gilbert JC, Budinger TF. Hypoxia in human gliomas: demonstration by PET with fluorine-18-fluoromisonidazole. J Nucl Med. 1992; 33(12):2133-7. [PubMed: 1334136]

5. Guo P, Hu B, Gu W, et al. Platelet-derived growth factor-B enhances glioma angiogenesis by stimulating vascular endothelial growth factor expression in tumor endothelia and by promoting pericyte recruitment. Am J Pathol. 2003; 162(4):1083-93. [PubMed: 12651601]

6. Winkler F, Kozin SV, Tong R, et al. Kinetics of vascular normalization by VEGFR2 blockade governs brain tumor response to radiation: Role of oxygenation, angiopoietin-1 and matrix metalloproteinases. Cancer Cell. 2004; 6:553-63. [PubMed: 15607960]

7. Zagzag D, Hooper A, Friedlander DR, et al. In situ expression of angiopoietins in astrocytomas identifies angiopoietin-2 as an early marker of tumor angiogenesis. Exp Neurol. 1999; 159(2):391400. [PubMed: 10506510] 
8. Carmeliet P, Jain RK. Angiogenesis in cancer and other diseases. Nature. 2000; 407(6801):249-57. [PubMed: 11001068]

9. Holash J, Maisonpierre PC, Compton D, et al. Vessel cooption, regression, and growth in tumors mediated by angiopoietins and VEGF. Science. 1999; 284(5422):1994-8. [PubMed: 10373119]

10. Shweiki D, Itin A, Soffer D, Keshet E. Vascular endothelial growth factor induced by hypoxia may mediate hypoxia-initiated angiogenesis. Nature. 1992; 359(6398):843-5. [PubMed: 1279431]

11. Batchelor TT, Duda DG, di Tomaso E, et al. Phase II study of cediranib, an oral pan-vascular endothelial growth factor receptor tyrosine kinase inhibitor, in patients with recurrent glioblastoma. J Clin Oncol. 2010; 28(17):2817-23. [PubMed: 20458050]

12. Friedman HS, Prados MD, Wen PY, et al. Bevacizumab alone and in combination with irinotecan in recurrent glioblastoma. J Clin Oncol. 2009; 27(28):4733-40. [PubMed: 19720927]

13. Batchelor TT, Sorensen AG, di Tomaso E, et al. AZD2171, a pan-VEGF receptor tyrosine kinase inhibitor, normalizes tumor vasculature and alleviates edema in glioblastoma patients. Cancer Cell. 2007; 11(1):83-95. [PubMed: 17222792]

14. Gerstner ER, Chen PJ, Wen PY, Jain RK, Batchelor TT, Sorensen G. Infiltrative patterns of glioblastoma spread detected via diffusion MRI after treatment with cediranib. Neuro Oncol. 2010; 12(5):466-72. [PubMed: 20406897]

15. de Groot JF, Fuller G, Kumar AJ, et al. Tumor invasion after treatment of glioblastoma with bevacizumab: radiographic and pathologic correlation in humans and mice. Neuro Oncol. 2010; 12(3):233-42. [PubMed: 20167811]

16. Mancuso MR, Davis R, Norberg SM, et al. Rapid vascular regrowth in tumors after reversal of VEGF inhibition. J Clin Invest. 2006; 116(10):2610-21. [PubMed: 17016557]

17. Ebos JM, Lee CR, Cruz-Munoz W, Bjarnason GA, Christensen JG, Kerbel RS. Accelerated metastasis after short-term treatment with a potent inhibitor of tumor angiogenesis. Cancer Cell. 2009; 15(3):232-9. [PubMed: 19249681]

18. Louis, DN.; Ohgaki, H.; Wiestler, OD.; Cavenee, WK. World Health Organization classification of tumors of the Central Nervous System. Lyon: IARC Press; 2007.

19. Zhu AX, Sahani DV, Duda DG, et al. Efficacy, safety, and potential biomarkers of sunitinib monotherapy in advanced hepatocellular carcinoma: a phase II study. J Clin Oncol. 2009; 27(18): 3027-35. [PubMed: 19470923]

20. Pennacchietti S, Michieli P, Galluzzo M, Mazzone M, Giordano S, Comoglio PM. Hypoxia promotes invasive growth by transcriptional activation of the met protooncogene. Cancer Cell. 2003; 3(4):347-61. [PubMed: 12726861]

21. Rubenstein JL, Kim J, Ozawa T, et al. Anti-VEGF antibody treatment of glioblastoma prolongs survival but results in increased vascular cooption. Neoplasia. 2000; 2(4):306-14. [PubMed: 11005565]

22. Kunkel $\mathrm{P}$, Ulbricht $\mathrm{U}$, Bohlen $\mathrm{P}$, et al. Inhibition of glioma angiogenesis and growth in vivo by systemic treatment with a monoclonal antibody against vascular endothelial growth factor receptor-2. Cancer Res. 2001; 61(18):6624-8. [PubMed: 11559524]

23. Du R, Lu KV, Petritsch C, et al. HIF1alpha induces the recruitment of bone marrow-derived vascular modulatory cells to regulate tumor angiogenesis and invasion. Cancer Cell. 2008; 13(3): 206-20. [PubMed: 18328425]

24. Casanovas O, Hicklin DJ, Bergers G, Hanahan D. Drug resistance by evasion of antiangiogenic targeting of VEGF signaling in late-stage pancreatic islet tumors. Cancer Cell. 2005; 8(4):299_ 309. [PubMed: 16226705]

25. Paez-Ribes M, Allen E, Hudock J, et al. Antiangiogenic therapy elicits malignant progression of tumors to increased local invasion and distant metastasis. Cancer Cell. 2009; 15(3):220-31. [PubMed: 19249680]

26. Narayana A, Golfinos JG, Fischer I, et al. Feasibility of using bevacizumab with radiation therapy and temozolomide in newly diagnosed high-grade glioma. Int J Radiat Oncol Biol Phys. 2008; 72(2):383-9. [PubMed: 18793954]

27. Norden AD, Young GS, Setayesh K, et al. Bevacizumab for recurrent malignant gliomas: efficacy, toxicity, and patterns of recurrence. Neurology. 2008; 70(10):779-87. [PubMed: 18316689] 
28. Fischer I, Cunliffe CH, Bollo RJ, et al. High-grade glioma before and after treatment with radiation and Avastin: initial observations. Neuro Oncol. 2008; 10(5):700-8. [PubMed: 18697955]

29. Shojaei F, Wu X, Malik AK, et al. Tumor refractoriness to anti-VEGF treatment is mediated by CD11b+Gr1+ myeloid cells. Nat Biotechnol. 2007; 25(8):911-20. [PubMed: 17664940]

30. Steidl C, Lee T, Shah SP, et al. Tumor-associated macrophages and survival in classic Hodgkin's lymphoma. N Engl J Med. 2010; 362(10):875-85. [PubMed: 20220182] 


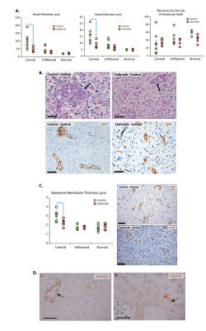

Figure 1. Vascular changes after cediranib treatment

A: Quantification of vessel perimeters and diameters in central and infiltrative areas of tumor as well as normal brain tissue in control patients or after cediranib by CD31 immunohistochemistry shows broad variability in central areas of control cases and a narrower spectrum after cediranib treatment. Cediranib-treated tumor vessels have a significantly smaller average perimeter and diameter in the central area $(\mathrm{p}=0.02)$, very close to that of normal brain capillaries. Quantification of microvascular density shows that cediranib treated tumors have a similar microvascular density in the central and infiltrating areas and that these are similar to the microvascular density of uninvolved brain. Microvascular density of control cases has a broader variation in the central and infiltrated areas. B: Microvascular proliferation typical of GBM vessels was abundant in all control cases (black arrow); however it was decreased in cediranib-treated patients (arrow points at the most abnormal vessel lining). Periodic and Schiff stain and CD31

immunohistochemistry highlight abnormal vascular morphology. C: Cediranib-treated tumor vessels in the central areas have a thinner basement membrane $(\mathrm{p}=0.03)$, similar to normal vessels (Collagen IV immunohistochemistry). Immunohistochemistry for Collagen IV shows marked thickening of the basement membranes in the central are of the control tumors and thinner basement membranes of the vessels in the cediranib treated tumors. D: Immunohistochemistry highlights expression of PDGFRbeta by the endothelial cells in diagnostic biopsy tissue from one of the patients (i-arrow). Autopsy tissue from the same patient after cediranib therapy (ii) displayed mostly PDGFRbeta negative vessels (endothelium unstained, red arrow) while the perivascular cells are positive for PDGFRbeta as expected for normal brain vessels (black arrow). Scale bar corresponds to $50 \mu \mathrm{m}$. 


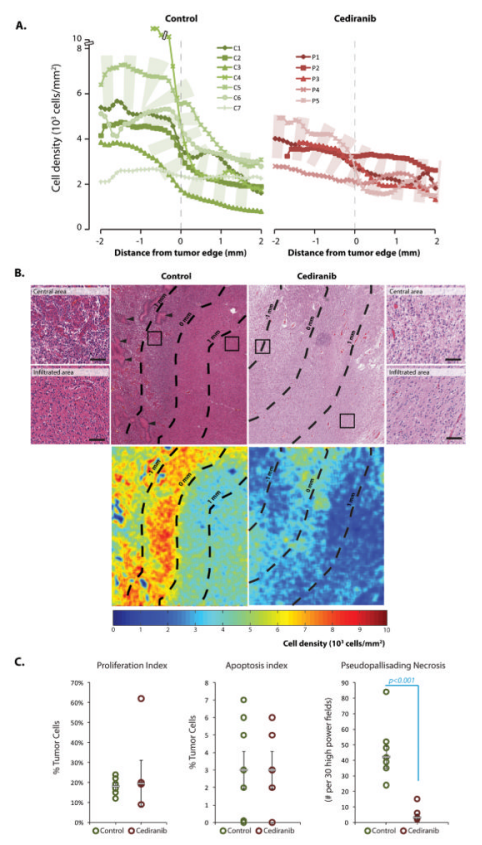

Figure 2. Cell density in recurrent glioblastoma (GBMs) after cediranib treatment

A: GBMs without previous antiangiogenic treatment (control) have a broad spectrum of cell density within the center of the tumor with a sharp drop in nuclear density in the infiltrating edge, where nuclear density is less variable. Cediranib treated patients show overall decreased cell density in the central area of the tumor compared to the patients not treated with antiangiogenic agent, with a more gradual and less steep drop of cell density in the infiltrating edge. B: Low power and high power (inserts) power hematoxylin \& eosin examination and nuclear density heat map reveal that central areas of the control cases have strikingly high nuclear density, abundant vascular proliferation (top left insert) and pseudopalisading necrosis (arrow heads). In cediranib-treated tumors infiltrating and central areas have a similar nuclear density (scale bar corresponds to $100 \mu \mathrm{m}$ ). C: Ki-67 index and apoptotic index in the central areas did not show difference proliferation rate or cell death between control cases and cediranib treated cases. Cediranib treated rGBMs showed significantly lower number of foci with pseudopalisading necrosis in the central area of the tumor (p-value<0.001). 


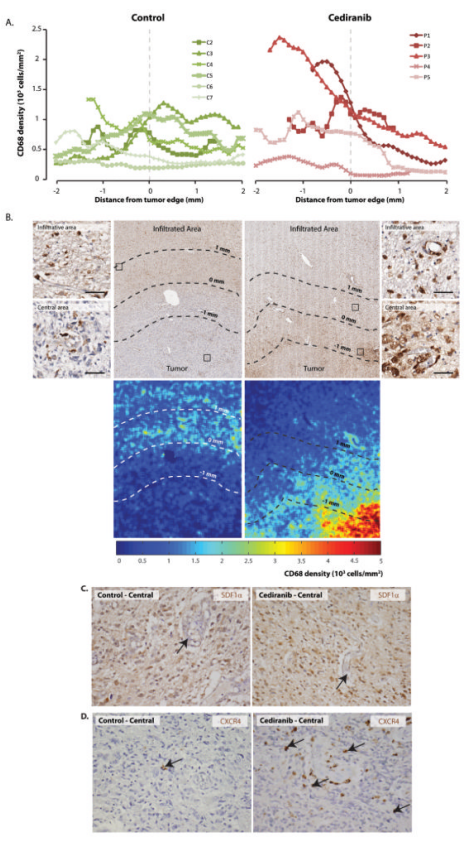

Figure 3. Tumor-infiltrating myeloid cells, SDF1 $\alpha$ and CXCR4 in cediranib treated rGBM A and B: Quantification of CD68 positive cells show similar numbers of macrophages in the central and infiltrating areas of control cases. Tumors from cediranib treated patients showed a trend towards higher numbers of macrophages in the central area, while the numbers of macrophages in the infiltrating areas are similar to control cases.

Immunohistochemistry for CD68 also reveals close association between macrophages and blood vessels (B, inserts). (scale bar corresponds to $50 \mu \mathrm{m})$. C: Although we did not observe difference in expression of SDF1 $\alpha$ (C) or CXCR4 (D) in tumor cells, control cases showed few CXCR4 tumor associated macrophages (3D left panel, arrow) while cediranib treated tumor contained many perivascular CXCR4 positive cells in the central area of the tumor (3D right panel, arrows). 
A.

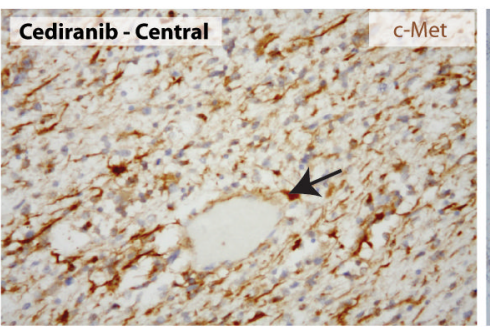

B.
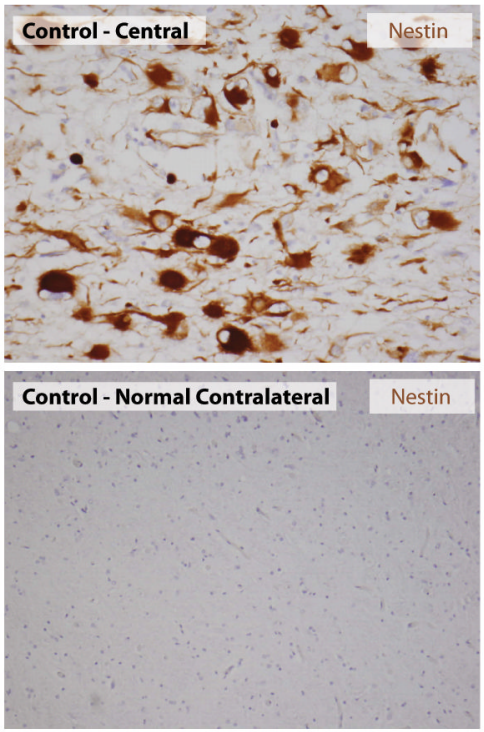
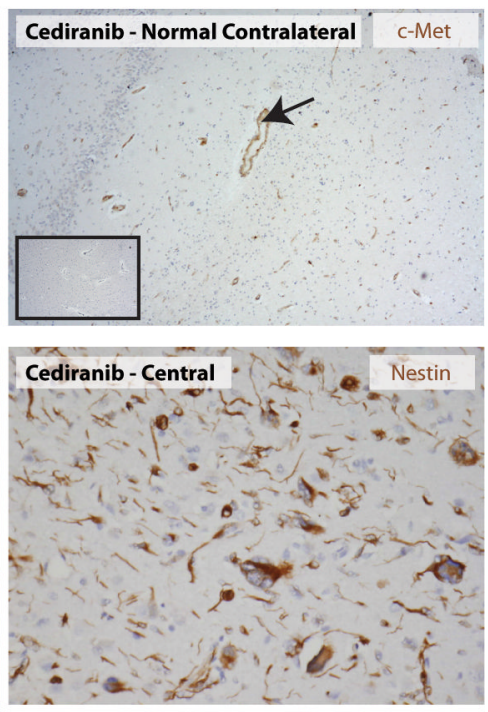

Cediranib - Normal Contralateral Nestin

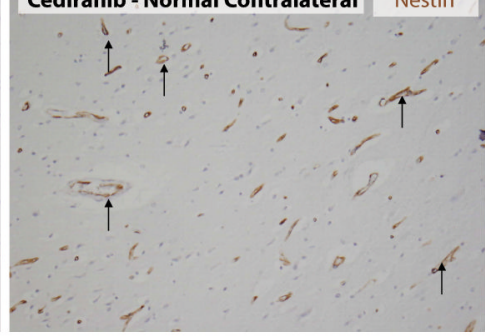

Figure 4. Expression of c-Met and Nestin in n cediranib treated rGBM

A: Diffuse c-Met expression was seen in several tumor cells as well as blood vessels (arrow) within the tumor areas after cediranib treatment. The blood vessels in the contra-lateral brain tissue (not involved by the tumor) of cediranib treated patients also expressed c-Met (right panel, arrow) while this was not seen in control autopsy cases (inset), where vessels in the brain tissue not involved by the tumor were negative. B: Nestin expression in the central area of the tumor (top left and right panel) is present in the tumor cells and vessels in both cediranib treated and control tumors. Of note, vessels were also nestin-positive in the normal contralateral part of the brain in cediranib treated patients (bottom right panel). This phenomenon was not seen in the control specimen (bottom left panel). 


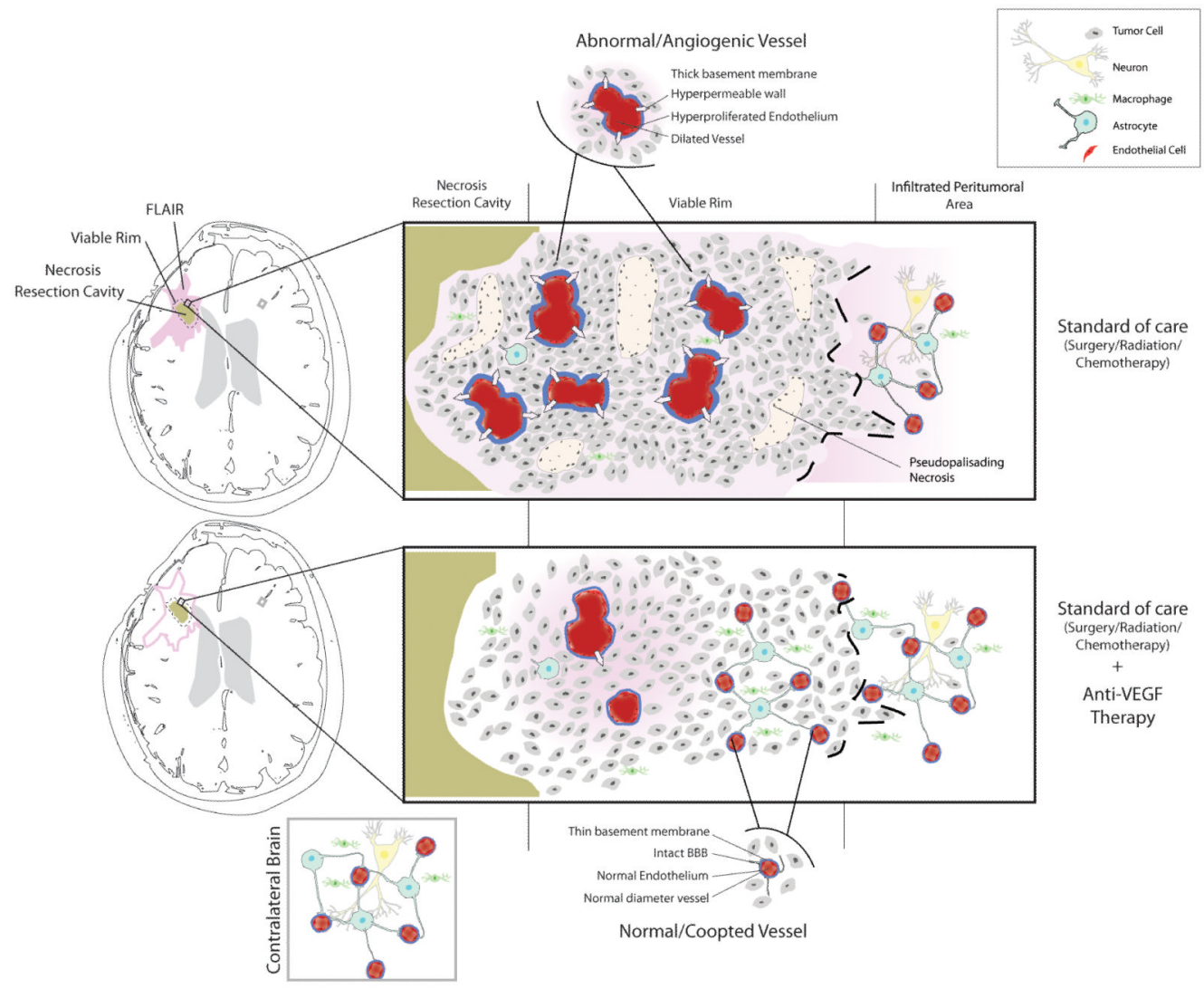

Figure 5. Model of changes in infiltrating behavior of recurrent GBM induced by cediranib The central area of a viable rGBM has high cell density with numerous abnormal leaky vessels, numerous foci of pseudopalisading necrosis and tumor cells which infiltrate surrounding normal brain (top panel). Anti-VEGF therapy (bottom panel) leads to decreased number of abnormal leaky vessels and lack of pseudopalisading necrosis. Cellular density in the central area is decreased while number of tumor cells in the infiltrating edge is similar to control cases. 


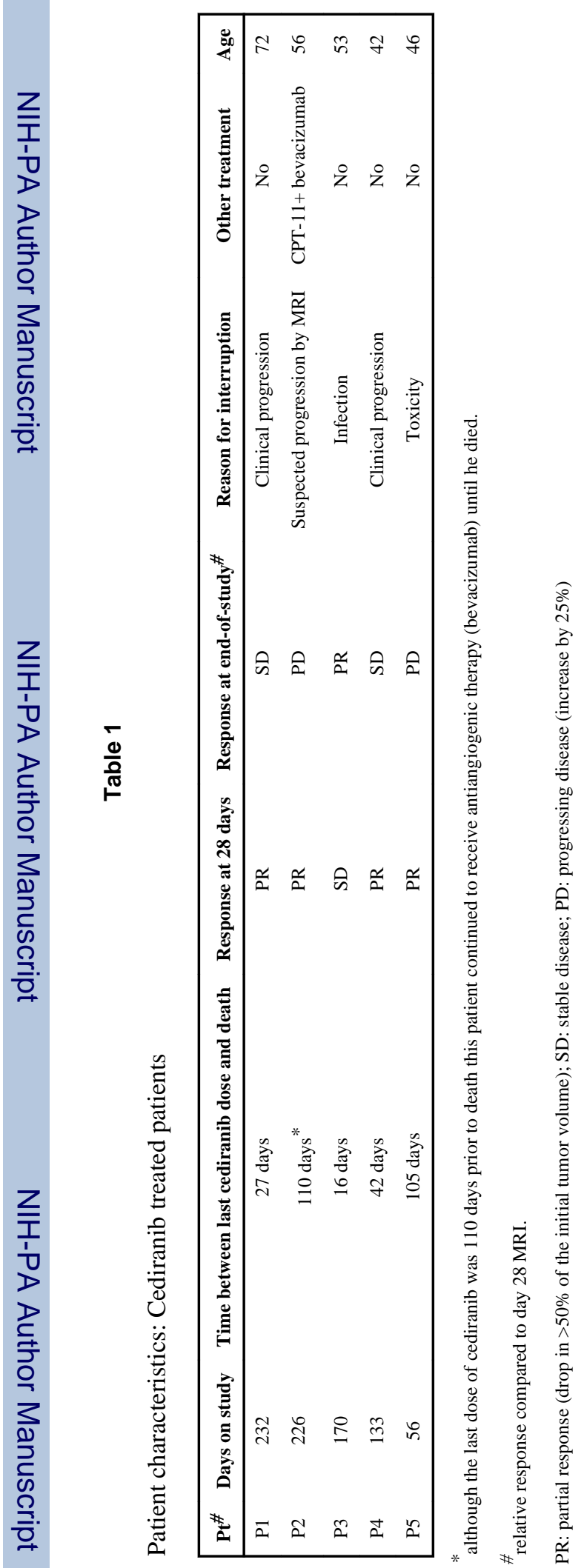

Cancer Res. Author manuscript; available in PMC 2012 January 1. 
Table 2

Patient characteristics: Patients with no antiangiogenic therapy (controls, after review of original charts and autopsy reports)

\begin{tabular}{|c|c|c|c|}
\hline $\mathbf{P t}^{\#}$ & Final Pathological Diagnosis & Treatment & Age \\
\hline $\mathrm{C} 1$ & GBM & Radiation (60Gy), gross total resection & 72 \\
\hline $\mathrm{C} 2$ & $\begin{array}{l}\text { GBM with prominent oligodendroglial } \\
\text { component }\end{array}$ & Radiation (dose unknown) and resection ${ }^{*}$ & 59 \\
\hline $\mathrm{C} 3$ & GBM & BCNU 2 cycles, gross total resection & 61 \\
\hline $\mathrm{C} 4$ & GBM - NF1 related & $\begin{array}{l}\text { Gross total resection, chemotherapy (Etoposide platinum, ARA-c), radiation } \\
\text { (50Gy) }\end{array}$ & 18 \\
\hline C5 & GBM & Resection post left carotid artery chemotherapy injection ${ }^{*}$ & 16 \\
\hline C6 & GBM & Multiple resections, radiation (55 Gy), chemotherapy (cisplatinum, BCNU) & 21 \\
\hline $\mathrm{C} 7$ & GBM & No Biopsies, No treatment- diagnosis confirmed by autopsy & 68 \\
\hline
\end{tabular}

* Original charts for patients C2 and C5 could not be obtained. Limited clinical data regarding their treatment were available from their autopsy reports. 